\title{
Electromagnetic Productions of the Hyperon and the Hypertriton Using Real and Virtual Photons
}

\author{
T. Mart $^{\mathrm{a}}$ \\ Departemen Fisika, FMIPA, Universitas Indonesia, Depok 16424, Indonesia
}

\begin{abstract}
The electromagnetic production of kaon-hyperon on the nucleon has been studied by means of a diagrammatic technique. All important resonances listed by the Particle Data Group up to spin 5/2 are included in this analysis. To extract the unknown coupling constants the new data from CLAS, SAPHIR, LEPS, and GRAAL collaborations have been used. In this paper we report on the preliminary results of this analysis. The available elementary model along with the modern nuclear wave functions have been used to analyze the process of photoas well as electroproduction of the hypertriton off the ${ }^{3} \mathrm{He}$. In this analysis we found that a proper treatment of Fermi motion is essential for describing the process, whereas the few available experimental data favor the assumption that the initial nucleon is off-shell and the final hyperon is on-shell. The influence of the higher partial waves in this process is also investigated. We also discuss the prospect of investigating kaon production off ${ }^{4} \mathrm{He}$.
\end{abstract}

\section{Introduction}

The present interest in the associated strangeness production is strongly motivated by the advancements in the accelerator and detector technologies. Compared to the relatively established pion photo- and electroproduction, the cross section of kaon electromagnetic production off the nucleon is three order of magnitude smaller. Therefore, in order to achieve reasonably accurate measurements, high intensity and continuous electron beams are inevitable. Together with the construction of modern detectors, this has been achieved in the last decades. As a consequence, significant improvement in the number and quality of experimental data, unprecedented before, have been also achieved recently.

The electromagnetic production of kaon is one of the important subjects in the investigation of the dynamics of the strangeness quantum number. It is obvious that the nonperturbative nature of QCD at these energies forces us to derive a number of approximations to QCD. One such attempt is the relativistic or relativized quark models. These models predict more excited states of nucleons than those found in experiments. Other "missing" states are suspected to weakly interact with the pion-nucleon state, a conventional mechanism used by the Particle Data Group (PDG) to determine the existence of the nucleon resonances. Thus, it is expected that these "missing resonances" may couple strongly to other channels, such as the $K \Lambda$ and $K \Sigma$ channels [1] or final states involving vector mesons.

In order to firmly investigate these missing resonances one needs a reliable elementary operator that can microscopically describe the process. There have been several approaches for this purpose, namely single-channel or multichannels analysis, multipoles approach, chiral quark calcu-

a e-mail: tmart@fisika.ui.ac.id lations, etc. Meanwhile, it is also important to remember that this operator is also required for the investigation of hypernuclear states. In this case, a fully covariant elementary operator has an obvious advantage, since the nuclear and hypernuclear wave functions are mostly constructed in a non-relativistic way and, therefore, the elementary operator should be constructed in a frame independent fashion. For this purpose, a single channel isobar model is very appropriate, since it provides a simple and covariant amplitude for use in the nuclear sector. The needs of this simple operator would become more obvious when we discuss the cpu-time required in the calculation of the hypernuclear states.

This paper is organized as follows: In Section 2 we will describe the construction of the elementary operator used in our investigation. The results of our analysis on the kaon photo- and electroproduction will be also presented in this Section. Section 3 will focus on the construction of the nuclear operator and the result of the analysis on the hypertriton production. In Section 6 we will conclude our findings.

\section{Elementary Operator}

\subsection{The Model}

The elementary process for kaon electroproduction off the nucleon can be written as

$$
e\left(k_{\mathrm{i}}\right)+N\left(p_{N}\right) \longrightarrow e^{\prime}\left(k_{\mathrm{f}}\right)+K\left(q_{K}\right)+Y\left(p_{Y}\right) .
$$

To phenomenologically describe this process we have used the Feynman diagrammatic technique. The appropriate diagrams for this process is depicted in Fig. 1. Such a technique has the advantage that the elementary amplitudes can be written in a completely covariant way and, 


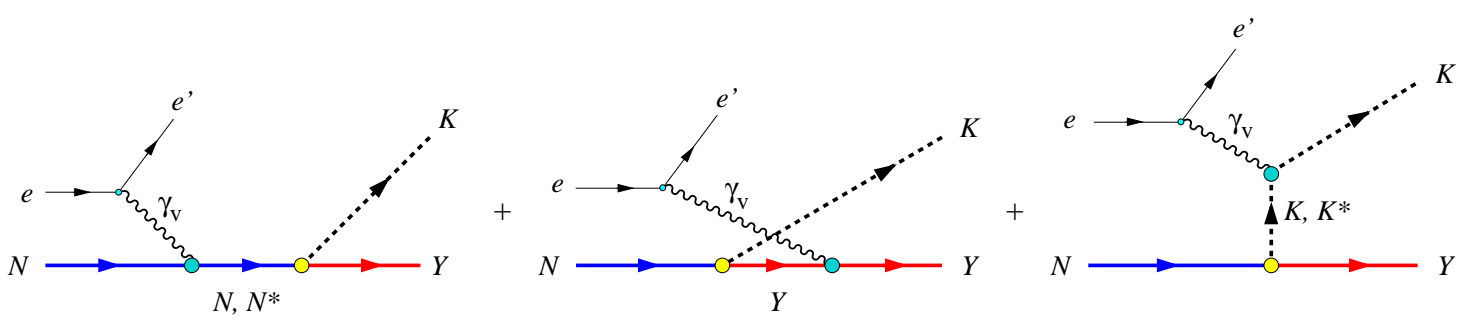

Fig. 1. Feynman diagrams for kaon electroproduction off the nucleon. Except contributions from the $N^{*}$ intermediate states, all diagrams are considered as the background terms. A contact diagram might be added to restore gauge invariance if we used the pseudovector coupling or included hadronic form factors.

therefore, the corresponding elementary operator can be expressed in a completely frame independent form. This is extremely important when we have to include Fermi motion in the nucleus.

As shown in Fig. 1, in this technique we assume that the electromagnetic interaction is mediated by only one photon exchange. The elementary transition operator can be written as

$$
M_{\mathrm{fi}}=\epsilon_{\mu} J^{\mu}=\bar{u}\left(\mathbf{p}_{Y}\right) \sum_{i=1}^{6} A_{i}\left(k^{2}, s, t, u\right) M_{i} u\left(\mathbf{p}_{N}\right) .
$$

where the virtual photon momentum $k=k_{\mathrm{i}}-k_{\mathrm{f}}$ and the Mandelstam variables are defined as

$$
s=\left(k+p_{N}\right)^{2}, t=\left(k-q_{K}\right)^{2}, u=\left(k-p_{Y}\right)^{2} .
$$

The gauge and Lorentz invariant matrices $M_{i}$ in Eq. (2) are given by

$$
\begin{aligned}
& M_{1}=\frac{1}{2} \gamma_{5}(\notin k-k \notin) \\
& M_{2}=\gamma_{5}\left[\left(2 q_{K}-k\right) \cdot \epsilon P \cdot k-\left(2 q_{K}-k\right) \cdot k P \cdot \epsilon\right] \\
& M_{3}=\gamma_{5}\left(q_{K} \cdot k \notin-q_{K} \cdot \epsilon k\right) \\
& M_{4}=i \epsilon_{\mu v \rho \sigma} \gamma^{\mu} q_{K}^{v} \epsilon^{\rho} k^{\sigma} \\
& M_{5}=\gamma_{5}\left(q_{K} \cdot \epsilon k^{2}-q_{K} \cdot k k \cdot \epsilon\right) \\
& M_{6}=\gamma_{5}\left(k \cdot \epsilon k-k^{2} \notin\right)
\end{aligned}
$$

with $P=\frac{1}{2}\left(p_{N}+p_{Y}\right)$ and $\epsilon_{\mu \nu \rho \sigma}$ represents the four dimensional Levi-Civita tensor with $\epsilon_{0123}=1$. The coefficient functions $A_{i}$ are obtained from Feynman diagrams shown in Fig. 1. All observables can be calculated from the coefficient functions $A_{i}$ by using the standard procedure [2]. Except the $N^{*}$, all diagrams contribute to the background terms. It is then clear that different models appear as the different use of the nucleon resonances $N^{*}$.

To relate the coupling constants among the various isospin channels, we first consider the hadronic vertices. Since the lambda is an $\mathrm{SU}(3)$ isosinglet, one obtains

$$
g_{K^{+} \Lambda p}=g_{K^{0} \Lambda n} .
$$

Similarly for the $N \rightarrow K^{*} \Lambda$ vertex

$$
g_{K^{*+} \Lambda p}^{V, T}=g_{K^{* 0} \Lambda n}^{V, T} .
$$

Therefore, Eqs. (10) and (11) couple the $\gamma+p \rightarrow K^{+}+\Lambda$ and $\gamma+n \rightarrow K^{0}+\Lambda$ channels.

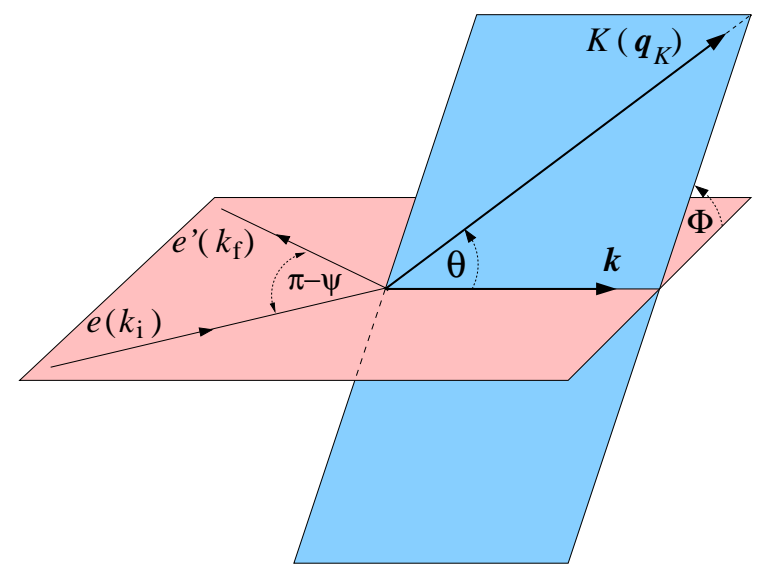

Fig. 2. Kinematic variables for kaon electroproduction on nucleons.

On the other hand, the sigma is an isovector. Therefore, the $N \rightarrow K \Sigma$ couplings are related by the Clebsch-Gordan coefficients coupling isospin 1 plus isospin $\frac{1}{2}$ to isospin $\frac{1}{2}$,

$$
g_{K^{+} \Sigma^{0} p}=-g_{K^{0} \Sigma^{0} n}=g_{K^{0} \Sigma^{+} p} / \sqrt{2}=g_{K^{+} \Sigma^{-} n} / \sqrt{2} .
$$

These relations are used to couple all four isospin channels in the $K \Sigma$ productions. Furthermore, in these processes the $\Delta$ resonance contributions with spin $1 / 2$ and $3 / 2$ are allowed. Their coupling constants are related by

$$
G_{\Delta} \equiv G_{K^{+} \Sigma^{0} \Delta^{+}}=-\sqrt{2} G_{K^{0} \Sigma^{+} \Delta^{+}}=G_{K^{0} \Sigma^{0} \Delta^{0}}=\sqrt{2} G_{K^{+} \Sigma^{-} \Delta^{0}}
$$

For unpolarized photons and hadrons the cross section of the real photon production can be simply written as

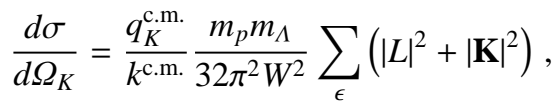

where the spin-independent $L$ and spin-dependent $\mathbf{K}$ amplitudes are related to the elementary amplitude $\epsilon^{\mu} J_{\mu}$ given by Eq. (2) through $\epsilon_{\mu} J^{\mu}=L+i \sigma \cdot \mathbf{K}$.

For unpolarized initial and final states of electrons and hadrons, the cross section of the virtual photon production can be written as

$$
\frac{d \sigma_{v}}{d \Omega_{K}}=\frac{d \sigma_{T}}{d \Omega_{K}}+\epsilon \frac{d \sigma_{L}}{d \Omega_{K}}+\epsilon \frac{d \sigma_{T T}}{d \Omega_{K}} \cos 2 \Phi
$$


Table 1. The resonances used in Kaon-Maid and in the present work.

\begin{tabular}{lcc}
\hline \hline State & Kaon-Maid & Present Work \\
\hline$S_{11}(1650)$ & $\sqrt{ }$ & $\sqrt{ }$ \\
$D_{15}(1675)$ & - & $\sqrt{ }$ \\
$D_{13}(1700)$ & - & $\sqrt{ }$ \\
$P_{11}(1710)$ & $\sqrt{ }$ & $\sqrt{ }$ \\
$P_{13}(1720)$ & $\sqrt{ }$ & $\sqrt{ }$ \\
$F_{15}(2000)$ & - & $\sqrt{ }$ \\
$D_{13}(2080)$ & $\sqrt{ }$ & $\sqrt{ }$ \\
$D_{15}(2200)$ & - & $\sqrt{ }$ \\
$D_{33}(1700)$ & - & $\sqrt{ }$ \\
$S_{31}(1900)$ & $\sqrt{ }$ & $\sqrt{ }$ \\
$F_{35}(1905)$ & - & $\sqrt{ }$ \\
$P_{31}(1910)$ & $\sqrt{ }$ & $\sqrt{ }$ \\
$P_{33}(1920)$ & - & $\sqrt{ }$ \\
$D_{35}(1930)$ & - & $\sqrt{ }$ \\
\hline \hline
\end{tabular}

$$
+\sqrt{\epsilon(1+\epsilon)} \frac{d \sigma_{L T}}{d \Omega_{K}} \cos \Phi,
$$

where $\Phi$ is the angle between the electron (scattering) and hadron reaction planes, and $\epsilon$ is the transverse polarization of the virtual photon

$$
\epsilon=\left(1-\frac{2 \mathbf{k}^{2}}{k^{2}} \tan ^{2}(\psi / 2)\right)^{-1},
$$

with $\psi$ the electron scattering angle. All kinematical variables given in Eqs. (15) and (16) are shown in Fig. 2.

The subscripts T, L, TT, and LT in Eq. (15) stand for transversely unpolarized, longitudinally polarized, transversely polarized, and interference between transversely and longitudinally polarized virtual photons. The individual terms may be expressed in terms of the amplitudes $A_{i}$, as given by Eqs. (4)-(9) [2].

One of the widely used phenomenological isobar models is Kaon-Maid. This model is constructed from gaugeinvariant background and resonances terms, whereas the background terms include the standard $s_{-}^{-}, u-$, and $t$-channel contributions along with a contact term required to restore gauge invariance after hadronic form factors have been introduced [3]. The resonance part consists of three nucleon resonances that have been found in the coupled-channels approach to decay into the $K \Lambda$ channel, i.e., the $S_{11}(1650)$, $P_{11}(1710)$, and $P_{13}(1720)$. Furthermore, the model also includes the $D_{13}(1895)$ state that is found to be important in the description of SAPHIR data [4].

We note that Kaon-Maid was fitted to a limited number of experimental data and therefore its predicting power is certainly very limited. Recent advancements in the accelerator and detector technologies in the last decades have paved the way to accurately measure the kaon production observables in a wide range of kinematics. It is then obvious that Kaon-Maid is no longer in the position to describe all these new data and a significant improvement of KaonMaid is inevitable.

In this paper, we propose a new model based on KaonMaid. For this purpose we add more resonances included

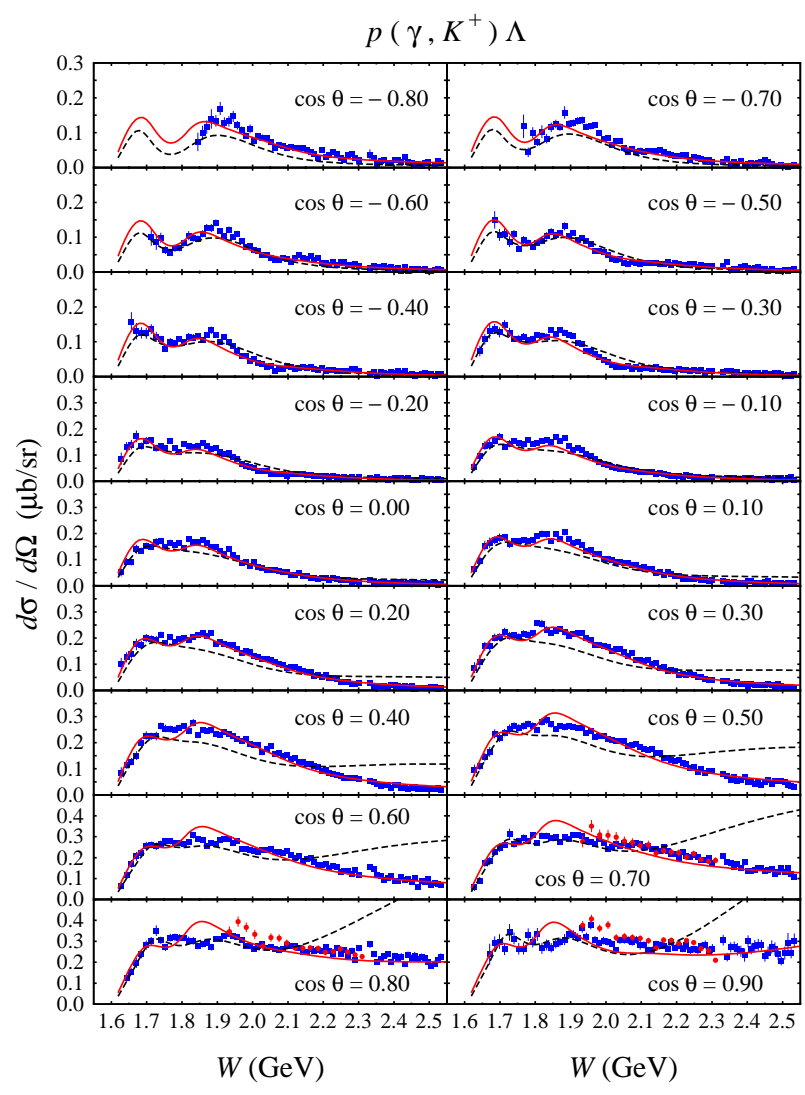

Fig. 3. Comparison between experimental data of the $\gamma+p \rightarrow$ $K^{+}+\Lambda$ kaon photoproduction differential cross section from CLAS (solid blue squares) [6] and LEPS (solid red circles) [11] with the model calculations from Kaon-Maid (dashed black lines) and the present calculation (solid red line).

in the process, because we want to include recent experimental data, i.e. cross sections as well as single and double polarization observables, with higher energies up to $2.5 \mathrm{GeV}$. A comparison between the resonances used in Kaon-Maid and those used in the present model is summarized in Table 1. Note that we use nucleon resonances up to spin $5 / 2$. The propagators and vertex factors for spin $3 / 2$ and 5/2 resonances are adopted from Ref. [5]. Furthermore, we maintain the use of hadronic form factors in the hadronic vertices of all diagrams, with the gauge prescription adopted from Haberzettl [3]. In the case of electroproduction we use the standard electromagnetic form factors for the Born terms, whereas the cutoffs of resonances are left as free parameters in the fitting process.

For photoproduction the fitted data include differential cross sections, recoiled $\Lambda$ polarizations, photon asymmetry, target asymmetry, and the beam-recoil polarization. For electroproduction we include the separated differential cross sections $\left(\sigma_{u}, \sigma_{T T}\right.$, and $\left.\sigma_{L T}\right)$ as well as the beamrecoil polarization data, both from the CLAS collaboration. Other older data were not used in our fits. 


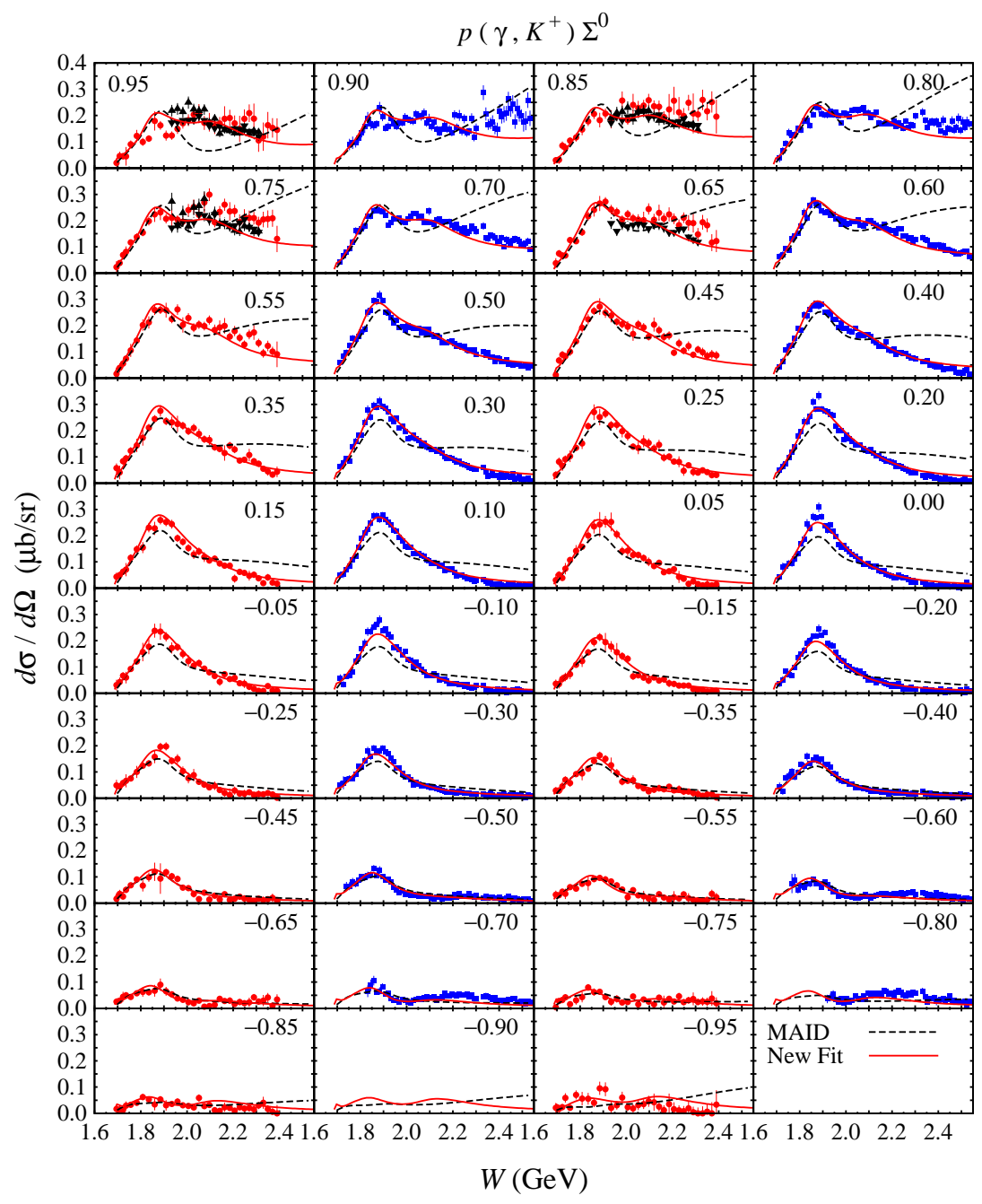

Fig. 4. As in Fig. 3, but for the $\gamma+p \rightarrow K^{+}+\Sigma^{0}$ channel. The solid black triangles show experimental data from the LEPS collaboration $[11,14]$. The cosines of the kaon scattering angles are shown in each panel.

\subsection{Results for Kaon Photoproduction}

For almost one decade, in the $\gamma+p \rightarrow K^{+}+\Lambda$ channel, there has been a problem of data consistency between the SAPHIR [4,7] and CLAS [6] data. Apparently, the problem just shows up in the differential cross sections at $W \approx$ $1900 \mathrm{MeV}$, where CLAS data are larger than SAPHIR ones. In Ref. [8] it has been shown, however, that the use of SAPHIR and CLAS data, individually or simultaneously, leads to quite different extracted photo-coupling parameters. To further investigate the differences of these two data sets, Ref. [9] has studied their statistical properties and it has been found that the CLAS data are in good agreement with the LEPS ones [11], whereas the SAPHIR data are coherently shifted down with respect to both CLAS and LEPS data, especially at forward kaon angles. In Ref. [10] it is suggested that measurements of the polarized total cross section $\sigma_{\mathrm{TT}^{\prime}}$ by the CLAS, LEPS, and MAMI collaborations are expected to help to solve this problem.

In this paper we only exclude the SAPHIR differential cross section data for the $\gamma+p \rightarrow K^{+}+\Lambda$ channel. Other data from SAPHIR, CLAS, LEPS, and GRAAL collaborations are included in our fits. Thus, we can avoid the long discussion on the data discrepancy problem as discussed above and focus on all isospin channels.

The result of our fit in this channel is shown in Fig. 3, where we compare the calculated differential cross sections of Kaon-Maid and present model with experimental data from the CLAS and LEPS collaborations. From this figure we can clearly see significant improvements are shown by the present calculation as compared to the KaonMaid. Only at the forward angles $\left(0.50 \leq \theta_{K}^{\text {c.m. }} \leq 0.9\right)$ we see that our new fit indicates apparent discrepancies at $W \approx 1.85 \mathrm{GeV}$. We suspect that this problem originates from the use of hadronic form factors. Such a problem was previously pointed out by Ref. [9]. It was found that the use of hadronic form factors leads to a strong suppression of the cross sections at forward angles and higher energies. In order to fit the data at this kinematics, the fit increase the cross sections at lower energies, which is compensated by the resonances at $W \approx 1.85 \mathrm{GeV}$. 
$19^{\text {th }}$ International IUPAP Conference on Few-Body Problems in Physics

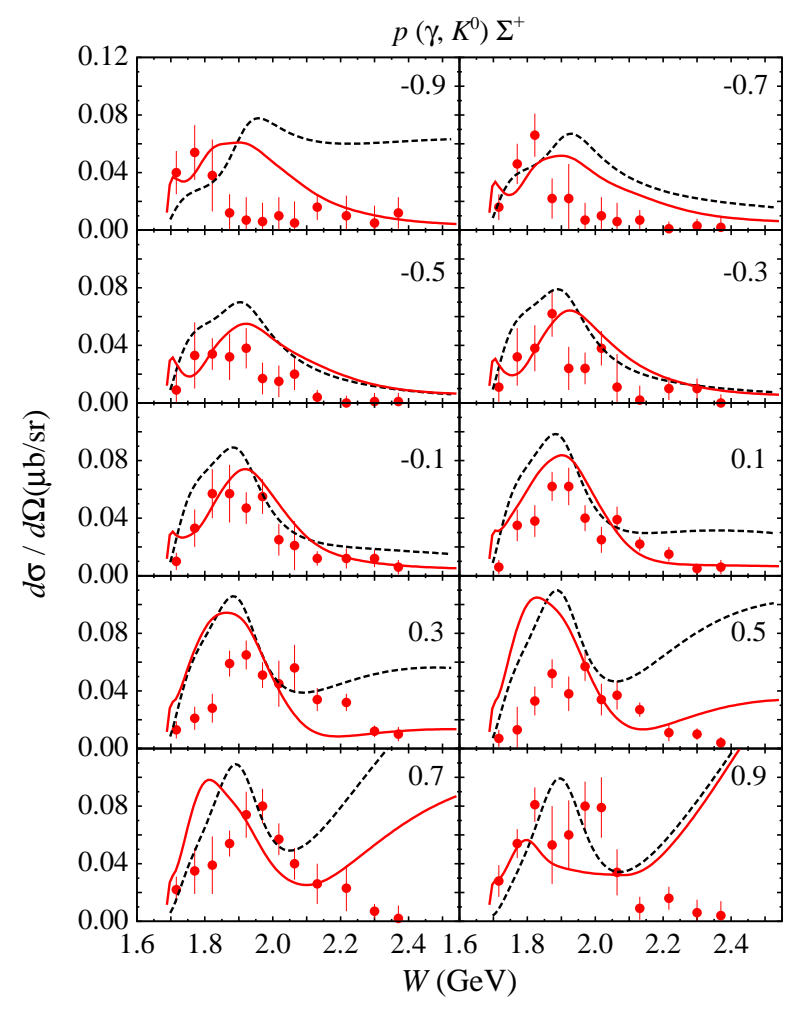

Fig. 5. As in Fig. 3, but for the $\gamma+p \rightarrow K^{0}+\Sigma^{+}$channel. Experimental data are from the SAPHIR collaboration [12].

For the $\gamma+p \rightarrow K^{+}+\Sigma^{0}$ channel, the result of our fit is shown in Fig. 4. It is clear from this figure that the results of our fits are much better than those of Kaon-Maid. Especially at higher energies, where the calculated cross sections of Kaon-Maid tend to diverge. This is understandable because Kaon-Maid does not have resonances with masses equal to or larger than $2.0 \mathrm{GeV}$. We observe that both SAPHIR and CLAS data indicate the need for a resonance, at least, at $W \approx 2.1 \mathrm{GeV}$.

Another important result found from this figure is that contributions from resonances with masses around $2.1 \mathrm{GeV}$ show up at the forward and very backward angles. Such an issue would be appropriately addressed for the future experiments of the LEPS collaboration at SPRING8, where kaon photoproduction at very forward and backward kaon angle are possible and can be measured accurately. At the present, LEPS data show an indication of resonance around $2.1 \mathrm{GeV}$. Nevertheless, by collecting more statistics one could single out the appropriate resonance at this energy unambiguously.

The photoproduction of $K^{0} \Sigma^{+}$reveals the fact that one could isolate contribution of resonances with masses around $1.9 \mathrm{GeV}$ by means of this isospin channel. This is clearly exhibited in Fig. 5. From this figure we can see that contribution of these resonances appear in almost all kaon angles except at the very forward regions.

Figure 5 also indicates the same deficiencies of both Kaon-Maid and the new model at high energies and forward directions in the $\gamma+p \rightarrow K^{0}+\Sigma^{+}$channel. This

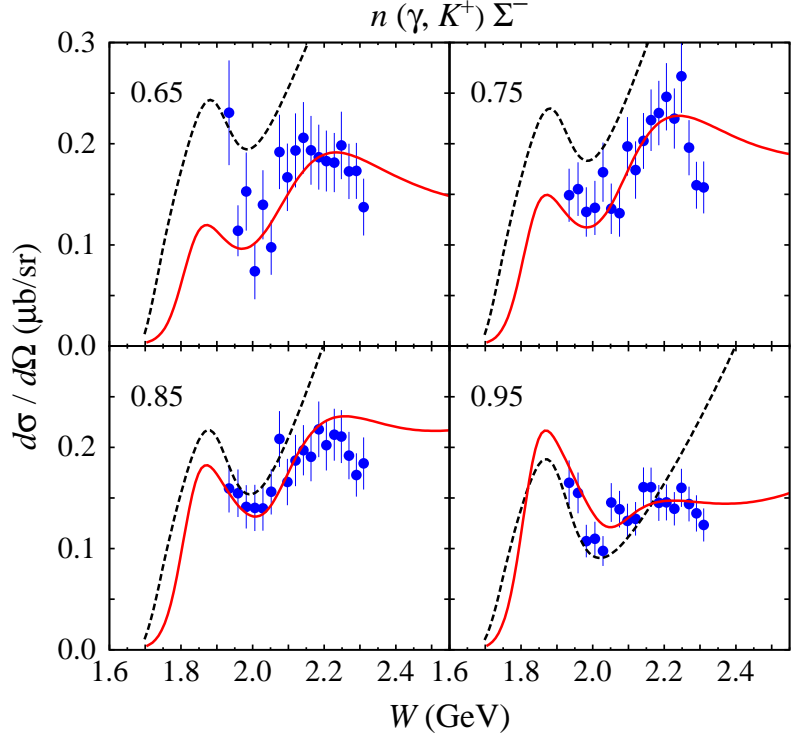

Fig. 6. As in Fig. 3, but for the $\gamma+n \rightarrow K^{+}+\Sigma^{-}$channel. Experimental data are from the LEPS collaboration [14].

problem is still investigated and will be reported in the future publication [13].

Experimental data for the production on neutron target are recently available from the LEPS collaboration [14]. These are the only neutron channel data available at present and, as a consequence, could provide a severe constraint to the models. The result of our fits in this channel is shown in Fig. 6. It is certainly interesting to see that the result of present work fits nicely the experimental data in this case. Furthermore, the data also indicate an important resonance contribution at $W \approx 2.2 \mathrm{GeV}$. Such a strong contribution at this energy is not observed in other isospin channels.

As we have pointed out in the beginning of this Section, we have performed our fits by including also single and double polarization observables data. Details of our findings for these observables will be reported in future [13].

\subsection{Results for Kaon Electroproduction}

Recently, the CLAS collaboration has released two set of electroproduction data bases consisting of a large number of experimental data on differential cross sections and polarization transfer $[15,16]$. Although we have used both data bases in our fits, we will only focus on the former in the following discussion.

In what follows we adopt the notation of Ref. [15]

$$
\begin{aligned}
\sigma_{U} & =\frac{d \sigma_{T}}{d \Omega_{K}}+\epsilon \frac{d \sigma_{L}}{d \Omega_{K}}, \\
\sigma_{T T} & =\frac{d \sigma_{T T}}{d \Omega_{K}}, \\
\sigma_{L T} & =\frac{d \sigma_{L T}}{d \Omega_{K}},
\end{aligned}
$$

where $\sigma_{U}$ refers to the unpolarized cross section. 


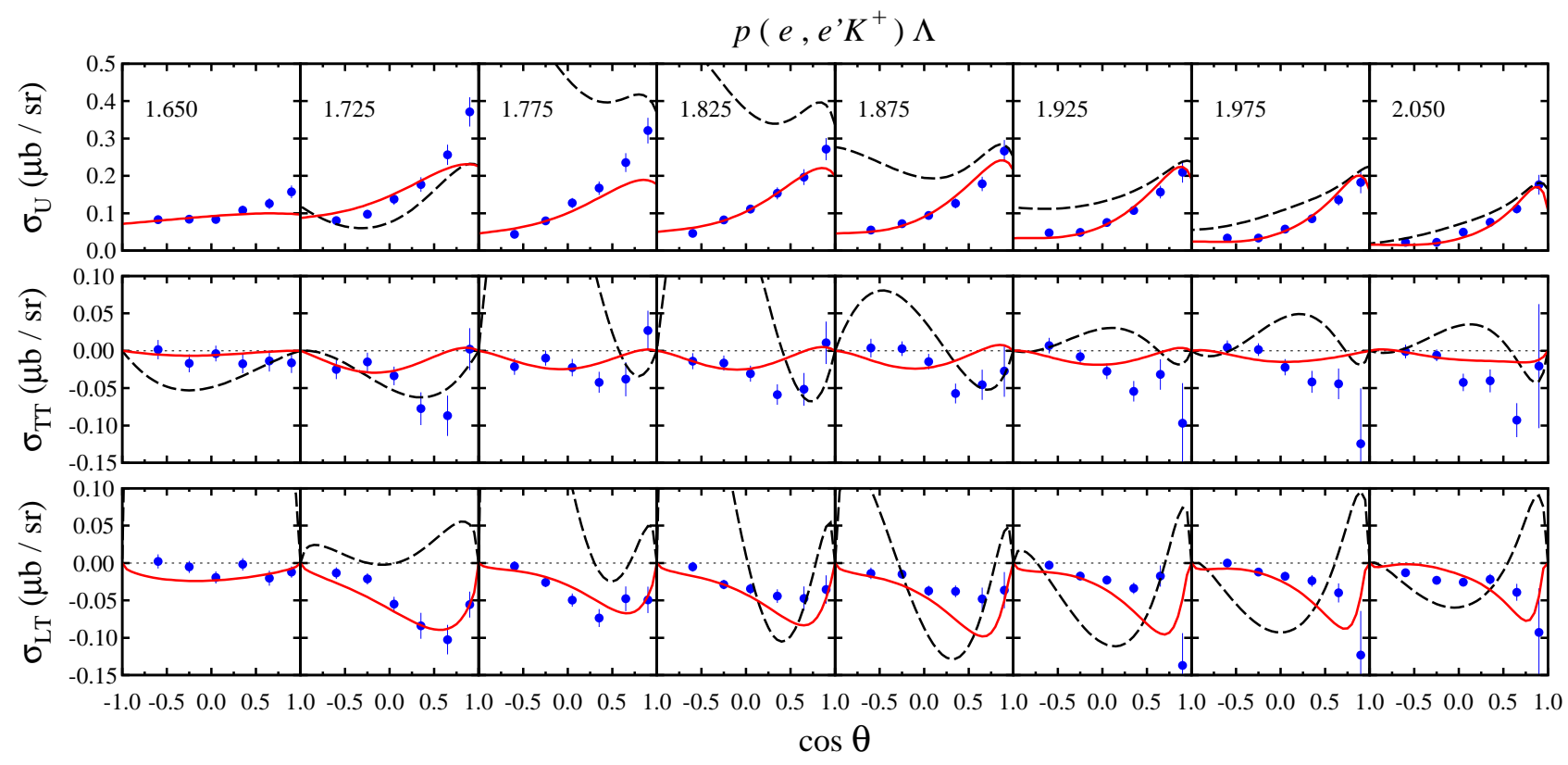

Fig. 7. Separated differential cross sections for kaon electroproduction $e+p \rightarrow e^{\prime}+K^{+}+\Lambda$ as a function of the kaon scattering angles for different total c.m. energies at $k^{2}=-0.65 \mathrm{GeV}^{2}$. Note that different total c.m. energies imply different virtual photon transverse polarization $\epsilon$. Experimental data are from the CLAS collaboration [15]. Notation of the curves is as in Fig. 3.

Results of the fit for the case of $K^{+} \Lambda$ and $K^{+} \Sigma^{0}$ electroproduction are shown in Fig. 7 and Fig. 9, respectively. It is obvious from the two figures that the present work provide substantial improvements on the Kaon-Maid model. Especially at low energies, where the prediction of Kaon-Maid enormously overestimate experimental data of the $\sigma_{U}$ and $\sigma_{T T}$ of the $e+p \rightarrow e^{\prime}+K^{+}+\Lambda$ channel. In general, the forward peaking behavior of $\sigma_{U}$ is nicely reproduced. Nevertheless, At low energies we observe that our new calculation slightly underestimate forward angles data. We suspect that this phenomenon has the common origin as in the case of photoproduction, i.e., the hadronic form factors. Since these form factors result in a strong suppression at forward angles and higher energies, the cross sections at lower energies must be raised up in order to reproduce experimental data at higher energies. The larger error bars at forward angles allow this process.

The calculated $\sigma_{T T}$ cross sections have mostly negative values as desired by the experimental data, while the calculated $\sigma_{L T}$ cross sections are all negative, in accordance with data. In both $\sigma_{T T}$ and $\sigma_{L T}$ the crossing behavior predicted by Kaon-Maid is not observed by the new data as well as the present calculation.

From Fig. 7 it is also apparent that there exist drastic changes in the cross section behavior at low energies. This happens in all three cross sections $\sigma_{U}, \sigma_{T T}$, and $\sigma_{L T}$ especially at $1.650 \leq W \leq 1.825 \mathrm{GeV}$. We have investigated the origin of this behavior and found that this is due to the huge contributions of the $S_{11}(1650)$ and $P_{13}(1720$ resonances in the case of electroproduction. Their contributions to the separated $d \sigma_{T} / d \Omega$ and $d \sigma_{L} / d \Omega$ cross sections at three different kaon angles are depicted in Fig. 8. It is clear from this figure that in the new fits these contributions

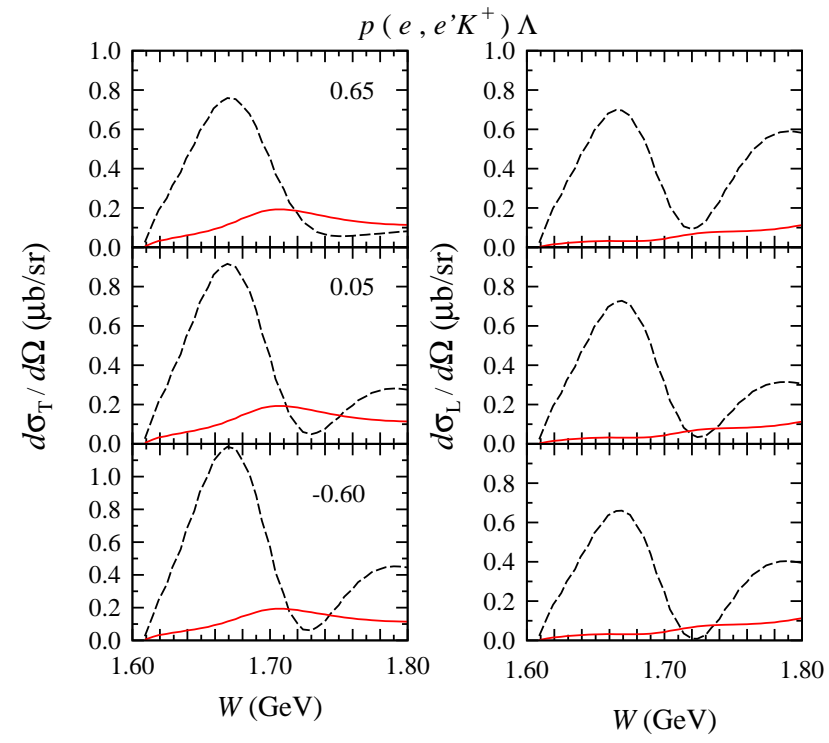

Fig. 8. Separated longitudinally and transversely unpolarized cross sections for the $e+p \rightarrow e^{\prime}+K^{+}+\Lambda$ channel as a function of the total c.m. energies at three different kaon angles.

are strongly suppressed in order to reproduce experimental data, which are of the order of $0.1 \mu \mathrm{b} / \mathrm{sr}$. At $W \approx 1.725$ $\mathrm{GeV}$ the cross sections show minima, which explain the drastic change at $1.725 \mathrm{GeV}$ in Fig. 7.

Results for the $e+p \rightarrow e^{\prime}+K^{+}+\Sigma^{0}$ channel are shown in Fig. 9. In this channel we observe almost the same behavior as in the case of $K^{+} \Lambda$ electroproduction, although we do not see the problem of hadronic form factors here. The larger error bars of $\sigma_{T T}$ in this channel is suspected to 


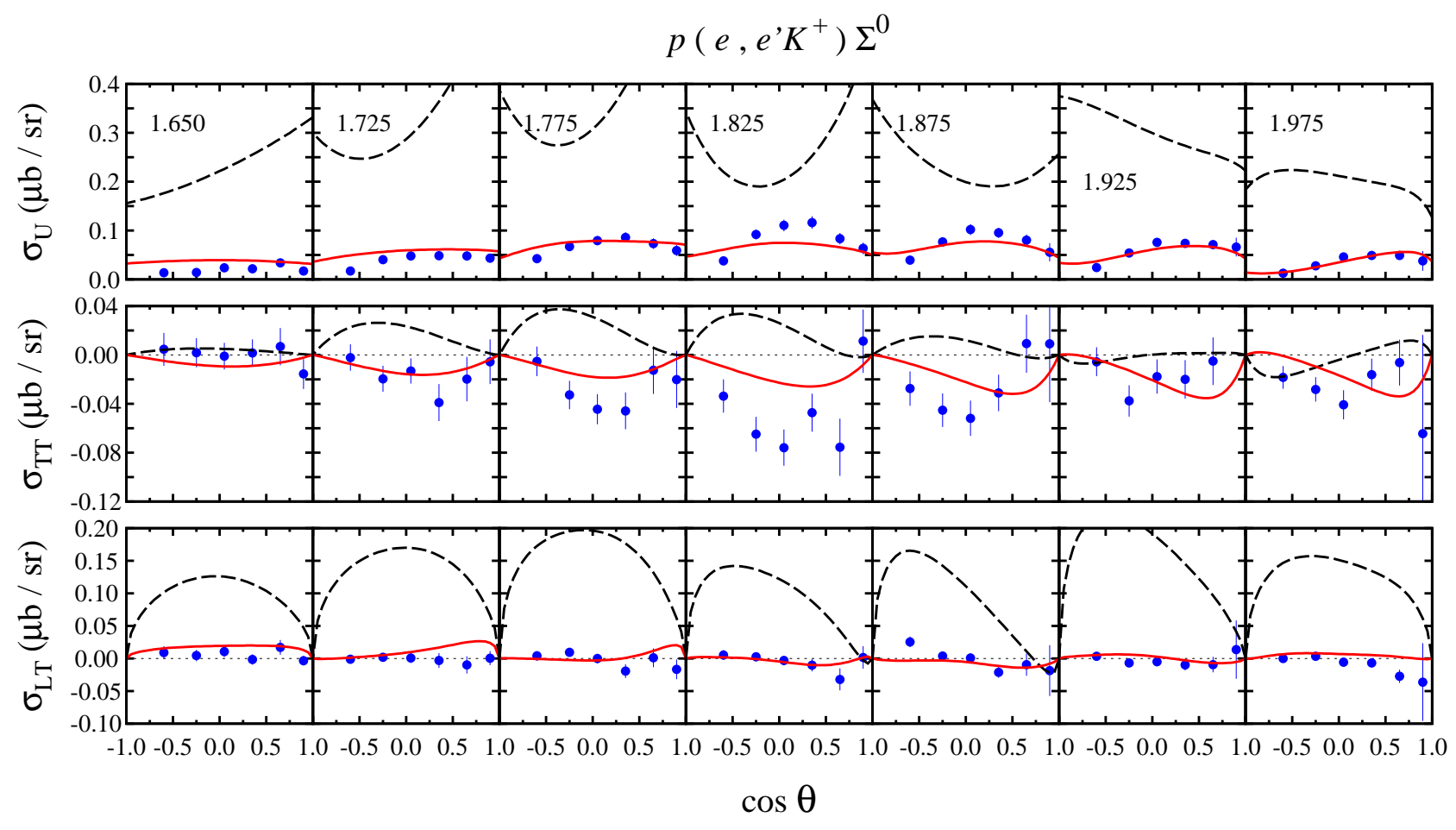

Fig. 9. As in Fig. 7, but for the $e+p \rightarrow e^{\prime}+K^{+}+\Sigma^{0}$ channel.

be the origin of the fit flexibility, which is indicated by the discrepancies between data and calculated cross sections at all energies.

The difference between this channel to the $K^{+} \Lambda$ one is also apparent here, i.e., in this channel the cross sections do not show the forward peaking behavior, a phenomenon that is also found in the case of photoproduction.

We also note that using these new data bases it is possible to separate $\sigma_{T}$ and $\sigma_{L}$ from the unpolarized cross sections for both isospin channels. However, since the error bars for $\sigma_{L}$ are significantly large, we will not discuss this topic in this paper. We will report a complete result in our future publication [13].

To conclude this section we would like to say that the separated cross sections of $\sigma_{L}$ and $\sigma_{T}$ are urgently required in order to shed more light on the small contribution of the $S_{11}(1650)$ resonance in the $K^{+} \Lambda$ isospin channel (see Fig. $8)$.

\section{Nuclear Operator}

The corresponding Feynman diagram for the hypertriton electroproduction on a ${ }^{3} \mathrm{He}$ target in an impulse approximation is displayed in Fig. 10. From this figure it is obvious that we can write the nuclear transition matrix element in the laboratory frame as

$$
\left\langle\mathrm{f}\left|J^{\mu}\right| \mathrm{i}\right\rangle=\sqrt{3} \int d^{3} \mathbf{p} d^{3} \mathbf{q} \Psi_{\mathrm{f}}^{*}\left(\mathbf{p}, \mathbf{q}^{\prime}\right) J^{\mu}\left(\mathbf{k}, \mathbf{p}_{N}, \mathbf{p}_{Y}\right) \Psi_{\mathrm{i}}(\mathbf{p}, \mathbf{q}),
$$

where the integrations are taken over the three-body momentum coordinates

$$
\mathbf{p}=\frac{1}{2}\left(\mathbf{k}_{2}-\mathbf{k}_{3}\right), \mathbf{q}=\mathbf{k}_{1},
$$

and the hyperon momentum in the hypertriton is given by

$$
\mathbf{q}^{\prime}=\mathbf{k}_{1}+\frac{2}{3} \mathbf{Q}
$$

with the momentum transfer $\mathbf{Q}=\mathbf{k}-\mathbf{q}_{K}$. The factor of $\sqrt{3}$ on the right hand side of Eq. (20) comes from the antisymmetry of the initial state. The derivation of this factor is given in Appendix B of Ref. [21].

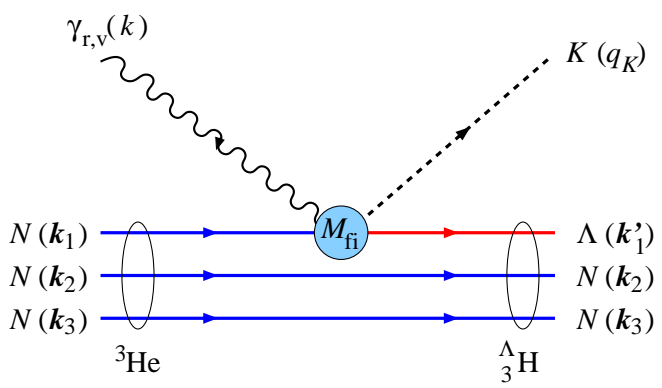

Fig. 10. Feynman diagram describing the process of the hypertriton electroproduction on a ${ }^{3} \mathrm{He}$ target in an impulse approximation.

The ${ }^{3} \mathrm{He}$ wave functions may be written as

$$
\Psi_{\mathrm{i}}(\mathbf{p}, \mathbf{q})=\sum_{\alpha} \phi_{\alpha}(p, q)\left|\left\{(L S) J,\left(l \frac{1}{2}\right) j\right\} \frac{1}{2} M_{\mathrm{i}}\right\rangle\left|\left(T \frac{1}{2}\right) \frac{1}{2} M_{t}\right\rangle
$$


Table 2. Quantum numbers and probabilities (in \%) of the ${ }^{3} \mathrm{He}$ and the hypertriton wave functions for the five leading partial waves. Note that there are 34 and 16 partial waves for the ${ }^{3} \mathrm{He}$ and hypertriton wave functions, respectively.

\begin{tabular}{ccccccccc}
\hline \hline$\alpha$ & $L$ & $S$ & $J$ & $l$ & $2 j$ & $2 T$ & $P\left({ }^{3} \mathrm{He}\right)$ & $P\left({ }_{\Lambda}^{3} \mathrm{H}\right)$ \\
\hline 1 & 0 & 0 & 0 & 0 & 1 & 1 & 44.580 & - \\
2 & 0 & 1 & 1 & 0 & 1 & 0 & 44.899 & 93.491 \\
3 & 2 & 1 & 1 & 0 & 1 & 0 & 2.848 & 5.794 \\
4 & 0 & 1 & 1 & 2 & 3 & 0 & 0.960 & 0.034 \\
5 & 2 & 1 & 1 & 2 & 3 & 0 & 0.189 & 0.027 \\
\hline \hline
\end{tabular}

where $\alpha=(L S J l j T)$ is introduced to shorten the notation, with $L, S$, and $T$ are the total angular momentum, spin, and isospin of the pair $(2,3)$, while for particle (1) the corresponding quantum numbers are labeled by $l, \frac{1}{2}$, and $\frac{1}{2}$, respectively. The hypertriton wave functions can also be written in the form of Eq. (23). The quantum numbers $\alpha$ for all partial waves of both the nuclear wave functions are listed in Ref. [21]. In Table 2 we gives only the most important partial waves that significantly contribute to the photoand electroproduction processes.

The elementary operator $J^{\mu}=\left(J^{0}, \mathbf{J}\right)$ given in Eq. (2) can be recast in the form of

$$
\begin{aligned}
J^{0}= & N\left\{i \mathcal{F}_{17} \mathbf{p}_{N} \cdot\left(\mathbf{p}_{Y} \times \mathbf{k}\right)+\left(\mathcal{F}_{2}-\mathbf{p}_{N} \cdot \mathbf{p}_{Y} \mathcal{F}_{17}\right) \boldsymbol{\sigma} \cdot \mathbf{k}\right. \\
& \left.+\left(\mathcal{F}_{6}+\mathbf{p}_{Y} \cdot \mathbf{k} \mathcal{F}_{17}\right) \boldsymbol{\sigma} \cdot \mathbf{p}_{N}+\left(\mathcal{F}_{10}+\mathbf{p}_{N} \cdot \mathbf{k} \mathcal{F}_{17}\right) \boldsymbol{\sigma} \cdot \mathbf{p}_{Y}\right\}
\end{aligned}
$$

and

$$
\begin{aligned}
\mathbf{J}= & -N\left[\left(\mathcal{F}_{1}+\mathcal{F}_{14} \mathbf{p}_{N} \cdot \mathbf{k}-\mathcal{F}_{15} \mathbf{p}_{Y} \cdot \mathbf{k}-\mathcal{F}_{16} \mathbf{p}_{N} \cdot \mathbf{p}_{Y}\right) \boldsymbol{\sigma}\right. \\
& +\boldsymbol{\sigma} \cdot \mathbf{k}\left\{\left(\mathcal{F}_{3}-\mathbf{p}_{N} \cdot \mathbf{p}_{Y} \mathcal{F}_{18}\right) \mathbf{k}+\left(\mathcal{F}_{4}-\mathcal{F}_{14}\right.\right. \\
& \left.\left.-\mathbf{p}_{N} \cdot \mathbf{p}_{Y} \mathcal{F}_{19}\right) \mathbf{p}_{N}+\left(\mathcal{F}_{5}+\mathcal{F}_{15}-\mathbf{p}_{N} \cdot \mathbf{p}_{Y} \mathcal{F}_{20}\right) \mathbf{p}_{Y}\right\} \\
& +\boldsymbol{\sigma} \cdot \mathbf{p}_{N}\left\{\left(\mathcal{F}_{7}+\mathcal{F}_{14}+\mathbf{p}_{Y} \cdot \mathbf{k} \mathcal{F}_{18}\right) \mathbf{k}+\left(\mathcal{F}_{8}\right.\right. \\
& \left.\left.+\mathbf{p}_{Y} \cdot \mathbf{k} \mathcal{F}_{19}\right) \mathbf{p}_{N}+\left(\mathcal{F}_{9}+\mathcal{F}_{16}+\mathbf{p}_{Y} \cdot \mathbf{k} \mathcal{F}_{20}\right) \mathbf{p}_{Y}\right\} \\
& +\boldsymbol{\sigma} \cdot \mathbf{p}_{Y}\left\{\left(\mathcal{F}_{11}+\mathcal{F}_{15}+\mathbf{p}_{N} \cdot \mathbf{k} \mathcal{F}_{18}\right) \mathbf{k}+\left(\mathcal{F}_{12}+\mathcal{F}_{16}\right.\right. \\
& \left.\left.+\mathbf{p}_{N} \cdot \mathbf{k} \mathcal{F}_{19}\right) \mathbf{p}_{N}+\left(\mathcal{F}_{13}+\mathbf{p}_{N} \cdot \mathbf{k} \mathcal{F}_{20}\right) \mathbf{p}_{Y}\right\} \\
& +i\left\{-\mathcal{F}_{14} \mathbf{p}_{N} \times \mathbf{k}-\mathcal{F}_{15} \mathbf{p}_{Y} \times \mathbf{k}+\mathcal{F}_{16} \mathbf{p}_{N} \times \mathbf{p}_{Y}\right. \\
& \left.\left.+\mathbf{p}_{N} \cdot\left(\mathbf{p}_{Y} \times \mathbf{k}\right)\left(\mathcal{F}_{18} \mathbf{k}+\mathcal{F}_{19} \mathbf{p}_{N}+\mathcal{F}_{20} \mathbf{p}_{Y}\right)\right\}\right]
\end{aligned}
$$

For the purpose of calculating the observables it is useful to rewrite the elementary operator in the form of a matrix $[j]$, through the relation $J^{\mu}=[\sigma][j]$, i.e.,

$$
J^{\mu}=\left(1, \sigma_{x}, \sigma_{y}, \sigma_{z}\right)\left(\begin{array}{llll}
j_{00} & j_{x 0} & j_{y 0} & j_{z 0} \\
j_{0 x} & j_{x x} & j_{y x} & j_{z x} \\
j_{0 y} & j_{x y} & j_{y y} & j_{z y} \\
j_{0 z} & j_{x z} & j_{y z} & j_{z z}
\end{array}\right),
$$

where the individual components are given in Appendix C of Ref. [21].

Since the hypertriton has isospin 0 , we may drop the isospin part of the wave functions. By inserting the two nuclear wave functions in Eq. (20) and writing symbolically $\mathrm{m}=\left(m_{L} m_{S} m_{l} m_{s} m_{J} m_{j}\right)$ for the sake of brevity, we can recast the transition matrix element in the form of

$$
\begin{aligned}
& \left\langle\mathrm{f}\left|J^{\mu}\right| \mathrm{i}\right\rangle= \\
& \sqrt{6} \sum_{\alpha, \alpha^{\prime}} \sum_{{\mathrm{m}, m^{\prime}}^{\prime}} \sum_{n, m_{n}}\left(L m_{L} S m_{S} \mid J m_{J}\right)\left(L m_{L} S m_{S} \mid J^{\prime} m_{J^{\prime}}\right) \\
& \times\left(l m_{l} \frac{1}{2} m_{s} \mid j m_{j}\right)\left(l^{\prime} m_{l^{\prime}} \frac{1}{2} m_{s^{\prime}} \mid j^{\prime} m_{j^{\prime}}\right)\left(J m_{J} j m_{j} \mid \frac{1}{2} M_{\mathrm{i}}\right) \\
& \times\left(J^{\prime} m_{J^{\prime}} j^{\prime} m_{j^{\prime}} \mid \frac{1}{2} M_{\mathrm{f}}\right)\left(\frac{1}{2}-m_{s^{\prime}} \frac{1}{2} m_{s} \mid n m_{n}\right) \\
& \times(-1)^{n-\frac{1}{2}-m_{s^{\prime}}} \delta_{L L^{\prime}} \delta_{m_{L} m_{L^{\prime}}} \delta_{S S^{\prime}} \delta_{m_{S} m_{S^{\prime}}} \delta_{T 0} \\
& \times \int p^{2} d p d^{3} \mathbf{q} \phi_{\alpha^{\prime}}\left(p, q^{\prime}\right) \phi_{\alpha}(p, q) Y_{m_{l^{\prime}}}^{l^{\prime}}\left(\hat{\mathbf{q}}^{\prime}\right) Y_{m_{l}}^{l}(\hat{\mathbf{q}})\left[j^{\mu}\right]_{m_{n}}^{(n)} .
\end{aligned}
$$

Note that the elementary operator $\left[j^{\mu}\right]_{m_{n}}^{(n)}$ is completely frame independent, since it is independent from the frame where $\epsilon^{\mu}$ and $\sigma^{(n)}$ are defined. Hence, by summing and averaging over the nuclear spins we can construct the spin averaged Lorentz tensor

$$
W^{\mu \nu}=\frac{1}{2} \sum_{M_{\mathrm{i}} M_{\mathrm{f}}}\left\langle\mathrm{f}\left|J^{\mu}\right| \mathrm{i}\right\rangle\left\langle\mathrm{f}\left|J^{\nu}\right| \mathrm{i}\right\rangle^{*},
$$

with which we can write the nuclear structure functions as

$$
\begin{aligned}
W_{\mathrm{T}} & =\frac{1}{4 \pi}\left(W_{x x}+W_{y y}\right), \\
W_{\mathrm{L}} & =\frac{1}{4 \pi} W_{00}, \\
W_{\mathrm{TT}} & =\frac{1}{4 \pi}\left(W_{x x}-W_{y y}\right), \\
W_{\mathrm{LT}} & =\frac{1}{4 \pi}\left(W_{0 x}+W_{x 0}\right) .
\end{aligned}
$$

The cross sections are conventionally measured in the c.m. system. In this frame of reference the individual cross sections are given by

$$
\frac{d \sigma_{i}}{d \Omega}=\frac{e^{2}}{4 \pi} \frac{q_{K} M^{3}{ }_{\mathrm{He}} M_{{ }^{3} \mathrm{H}}}{\left(W^{2}-M_{{ }^{\mathrm{He}}}^{2}\right) W} W_{i},
$$

with $i=\mathrm{T}, \mathrm{L}, \mathrm{TT}$, and LT.

\section{Results on the Hypertriton Production}

\subsection{The Numerical Results}

We first investigate the contribution of non-localities generated by Fermi motion in the initial and final nuclei. The exact treatment of Fermi motion is included in the integrations over the wave functions in Eq. (27). The local approximation can be carried out by freezing the operator at an average nucleon momentum (see Fig. 10)

$$
\left\langle\mathbf{k}_{1}\right\rangle=-\kappa \frac{A-1}{2 A} \mathbf{Q}=-\frac{\kappa}{3} \mathbf{Q},
$$




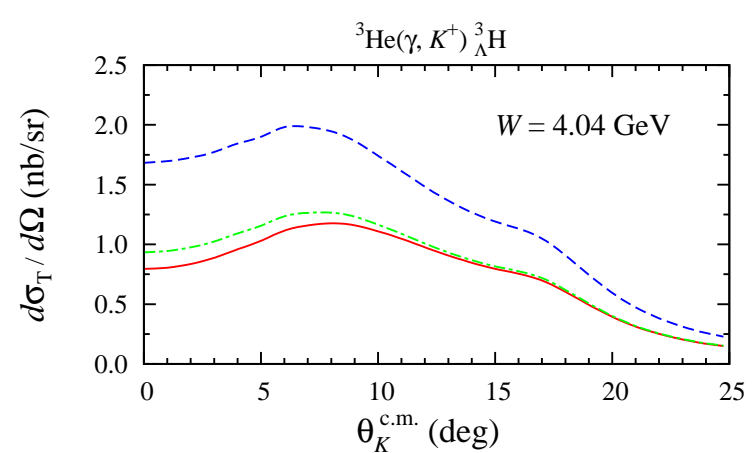

Fig. 11. Effects of Fermi motion on the differential cross section of the hypertriton photoproduction. The dashed curve is calculated from the "frozen nucleon" approximation $\left(\left\langle\mathbf{k}_{1}\right\rangle=0\right)$, the dash-dotted curve is obtained with an average momentum of $\left\langle\mathbf{k}_{1}\right\rangle=-\frac{1}{3} \mathbf{Q}$, while the solid curve shows the exact treatment of Fermi motion.

since $A=3$. For $\kappa=0$, Eq. (34) corresponds to the "frozen nucleon" approximation, whereas $\kappa=1$ yields the average momentum approximation.

The effect of these approximations on the calculated differential cross sections in the case of photoproduction is displayed in Fig. 11. It is obvious from this figure that the use of "frozen nucleon" approximation leads to significantly different results. Although the average momentum assumption can approximate the exact treatment of Fermi motion, for the sake of accuracy we will use the exact treatment of Fermi motion in the following discussion.

Since all baryons involved in the hypertriton productions are off-shell, whereas however, the elementary operator is constructed and fitted to experimental data with both initial nucleon and final hyperon are on-shell, off-shell assumption should be made here. For this purpose we propose four assumptions, i.e.,

1. both initial and final baryons are on-shell $\left[k_{1}^{0}=\left(m_{N}^{2}+\right.\right.$ $\left.\left.\mathbf{k}_{1}^{2}\right)^{1 / 2}, k_{1}^{\prime 0}=\left(m_{Y}^{2}+\mathbf{k}_{1}^{\prime 2}\right)^{1 / 2}\right]$

2. the initial nucleon is on-shell and the final hyperon is off-shell $\left[k_{1}^{0}=\left(m_{N}^{2}+\mathbf{k}_{1}^{2}\right)^{1 / 2}, k_{1}^{\prime 0}=k_{1}^{0}+k_{0}-E_{K}\right]$,

3. the initial nucleon is off-shell and the final hyperon is on-shell, $\left[k_{1}^{0}=k_{1}^{\prime 0}+E_{K}-k_{0}, k_{1}^{\prime 0}=\left(m_{Y}^{2}+\mathbf{k}_{1}^{\prime 2}\right)^{1 / 2}\right]$,

4. both initial and final baryons are off-shell. In this case the static approximation $k_{1}^{0}=m_{N}$ is used for the initial nucleon, while $k_{1}^{\prime 0}=k_{1}^{0}+k_{0}-E_{K}$.

These four different assumptions result in systematic variations of the differential cross sections as depicted in Fig. 12. From this figure we observe that the first assumption yields the smallest cross section, while the third assumption leads to the largest cross section. However, at higher $W$ the situation changes, the latter gives in fact the smallest cross sections. At $W=4.04 \mathrm{GeV}$ we estimate that experimental data at forward angles with about $10 \%$ error bars would be able to check these off-shell assumptions. For other kinematics (higher $W$ ) the cross section differences are presumably to small in view of the present technology [17].

Contribution of the missing resonance $D_{13}(1895)$ in the hypertriton production is also found to be significant, espe-

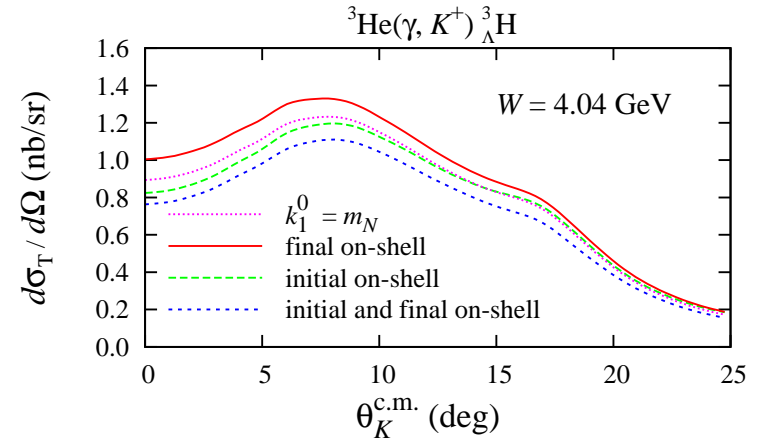

Fig. 12. The effect of different off-shell assumptions on the differential cross section calculated for the hypertriton photoproduction.

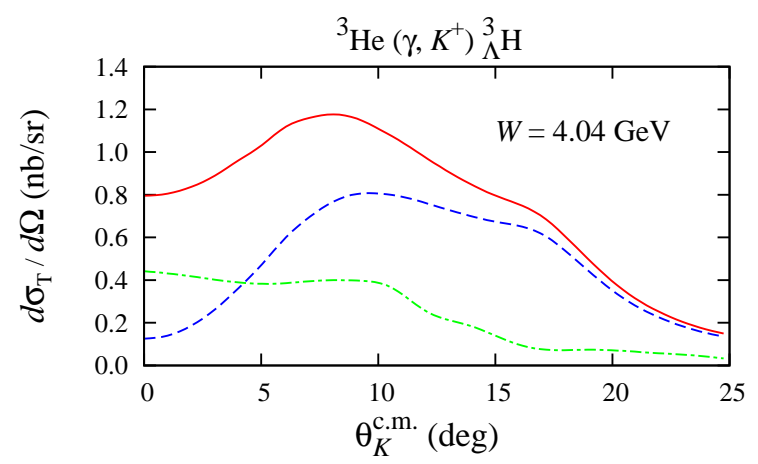

Fig. 13. Comparison between differential cross sections of hypertriton photoproduction obtained in the present work with the missing resonance $D_{13}(1895)$ included in the elementary operator (solid lines) and those obtained by excluding this resonance (dashed lines). Results from the previous work [22] which have been obtained by using different nuclear wave functions and different elementary operator [23] are shown by the dash-dotted lines.

cially at $W=4.04 \mathrm{GeV}$ (see Fig. 13). This results is understandable, because the energy corresponds to the elementary total c.m. energy $W_{\gamma p \rightarrow K \Lambda}=1.9 \mathrm{GeV}$, i.e., almost at the resonance pole position. As shown in Fig. 13, the effect gradually disappears at higher energies. We note that, due to the strong nuclear suppression at large kaon scattering angles, this effect also vanishes for $\theta_{K}^{\text {c.m. }}>25^{\circ}$. Therefore, $W \approx 4.04$ and $0^{\circ} \lesssim \theta_{K}^{\text {c.m. }} \lesssim 20^{\circ}$ represent an example of the recommended kinematics for the measurement of hypertriton photoproduction. This conclusion is apparently also supported by Fig. 11, where for this kinematics the variation in differential cross sections is found to be remarkable.

Off-shell effects are also found to be important in the case of electroproduction. This fact is clearly exhibited in Fig. 14, where we can see that the "final hyperon on-shell" assumption can nicely shift the cross section upward closer to the experimental data at $\theta_{K}^{\text {c.m. }}=2.7^{\circ}$ and $9.5^{\circ}$. Although this finding is in contrast to the phenomenon observed in the pion photoproduction off ${ }^{3} \mathrm{He}$ [18], it can be understood as follows: The hyperon binding energy in the hypertriton is much weaker than the binding energy of the nucleon in the ${ }^{3} \mathrm{He}$. Therefore, shifting the hyperon in the final state closer to its mass-shell moves the model closer to reality. 


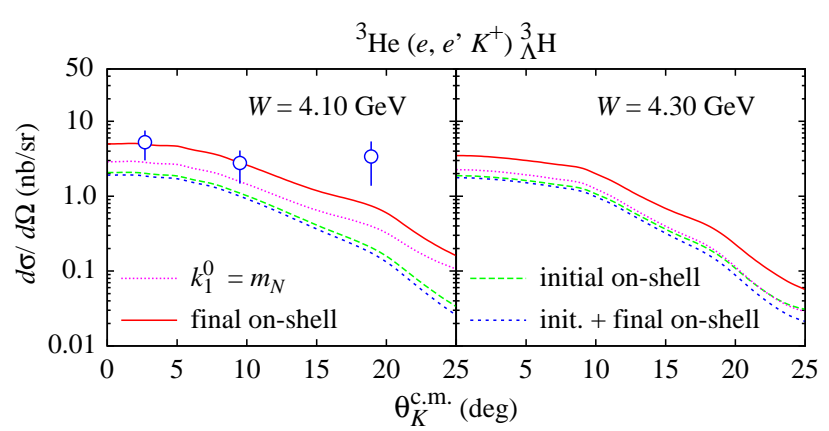

Fig. 14. The calculated cross section of the hypertriton electroproduction on ${ }^{3} \mathrm{He}$ compared with experimental data [17]. Different curves in both panels show different off-shell assumptions used in calculating the cross sections.

The experimental data point at $\theta_{K}^{\text {c.m. }}=18.9^{\circ}$ requires a special explanation. Although the elementary cross section at this kinematics slightly increases, the nuclear suppression from the two nuclear wave functions is sufficiently strong to reduce the cross sections at $\theta_{K}^{\text {c.m. }} \geq 10^{\circ}$.

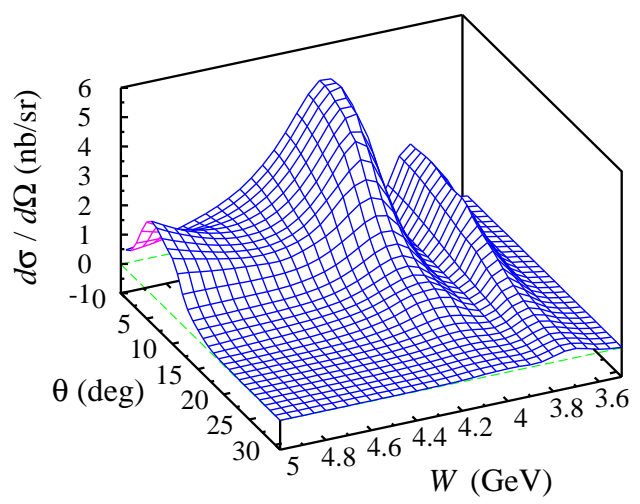

Fig. 15. Differential cross section of the hypertriton electroproduction on ${ }^{3} \mathrm{He}$ plotted as functions of the total c.m. energy and kaon scattering angle.

Since our investigations should be performed in a wide range of kinematics we also extend our analysis to higher energies and kaon scattering angles. The results however do not change our conclusion as described above. As an example, we have calculated the differential cross section of the hypertriton electroproduction as functions of $W$ and $\theta_{K}$, and show the plot in Fig. 15. From this figure it appears that the kinematical region where the experimental data exist $\left(W \approx 4.1 \mathrm{GeV}\right.$ and $\left.0^{\circ} \leq \theta_{K}^{\text {c.m. }} \leq 20^{\circ}\right)$ represent the region where the cross sections are considerably large.

\subsection{Effects of the Higher Partial Waves on the Numerical Accuracies}

It has been found that a full calculation by using all partial waves could cost a significantly long cpu-time. To understand this problem consider the integrations given in
Eq. (27). For the full calculation at every point of cross sections of interest integrations over $34 \times 20 \times 64 \times 30 \times 10 \approx$ $13 \times 10^{6}$ grid points should be carried out. The numerical computation becomes more challenging because the integrand consists of the elementary operator $\left[j^{\mu}\right]_{m_{n}}^{(n)}$ in the form of $4 \times 4$ complex-component matrix. For a more concrete example, the required cpu-time to calculate 961 points of the cross section for the three dimensional plot shown in Fig. 15 by using a single processor Pentium-4 PC would take about 11 days (15,344 min). Although this problem could be easily solved by using a parallel computing system, it would be appropriate to ask whether the use of all partial waves is really necessary for a sufficiently accurate numerical computation. The answer is shown in Fig. 16, where the required cpu-times to calculate the 961 points of the cross section for the full calculation and the three approximation, i.e., using $\alpha \leq 5,4$, and only $s$-waves, by means of a single processor $3 \mathrm{GHz}$ Pentium-4 PC are depicted.
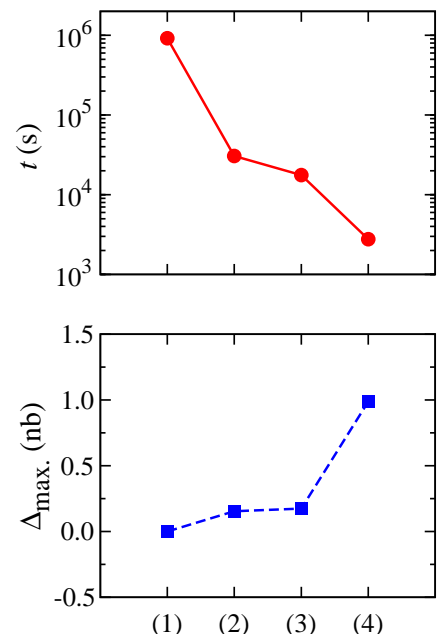

Fig. 16. The cpu-time $t$ required to make the three-dimensional plot shown in the Fig. 15 along with the maximum deviations from the full calculation for different approximations, i.e., using all partial waves (1), $\alpha \leq 5$ (2), $\alpha \leq 4$ (3), and only $s$-waves (4).

Clearly, the cpu-time is significantly reduced by a factor of 30 if we limit the partial waves up to $\alpha=5$, while the accuracy is still maintained up to about $0.15 \mathrm{nb} / \mathrm{sr}$. As a consequence, the required cpu-time to obtain the plot shown in Fig. 15 becomes less than 9 hours. If we used the partial waves with $\alpha \leq 4$, the cpu-time is reduced by a factor of about 60 , whereas the maximum deviation slightly increases to $0.17 \mathrm{nb} / \mathrm{sr}$. The use of only $s$-waves substantially reduces the cpu-time, i.e., by a factor of 300, but results in the largest deviation, almost $1 \mathrm{nb} / \mathrm{sr}$ at the top of the highest cross section peak. In view of the presently available experimental data (see Fig. 17), it is obvious that the latter provides a relatively poor approximation method for the hypertriton electroproduction, since the largest differential cross section is around $5 \mathrm{nb} / \mathrm{sr}$. 


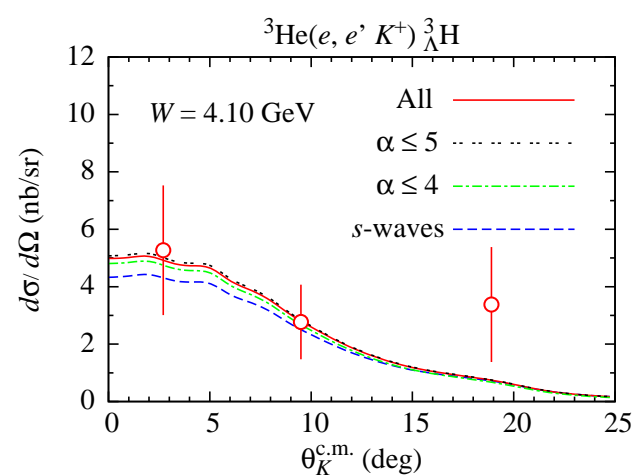

Fig. 17. Comparison between experimental data [17] and the calculation using all and specific numbers of partial waves.

We have also investigated the accuracy of the calculation if we limit the elementary amplitude to the nonrelativistic terms only [19]. Such an approximation was suggested, e.g. in Ref. [20], in order to reduce the numerical costs. We found that the omission of the relativistic terms in the elementary process amplitude could lead to a large deviation from the full calculation. Since no significant cpu-time reduction obtained by omitting these terms, a full calculation in this case is recommended.

\section{Future Investigation}

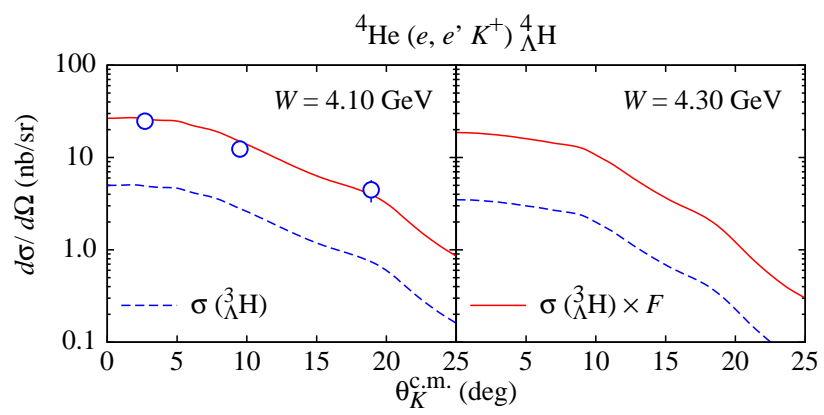

Fig. 18. (Left panel) Experimental data of the ${ }_{\Lambda}^{4} \mathrm{H}$ electroproduction cross section [17] compared with the calculated cross section of the ${ }_{\Lambda}^{3} \mathrm{H}$ electroproduction (dashed line) and with the scaled one (solid line). (Right panel) As in the left panel but for a different total c.m. energy.

Beside for the hypertriton electroproduction, experimental data have been also available [17] for the

$$
e+{ }^{4} \mathrm{He} \rightarrow e^{\prime}+K^{+}+{ }_{\Lambda}^{4} \mathrm{H}
$$

process. While the complete calculation is rather complicated, since it involves a relatively large number of partial waves, we can for the present estimate the cross section from our result for the hypertriton electroproduction.

First, it is obvious that since the hypertriton has isospin 0 , it does not have the $\alpha=1$ partial wave. As a consequence, the cross section becomes small because the $\alpha=$
1 partial wave is the leading term that significantly contributes to the amplitude. We estimate that by including this term, the amplitude could become roughly twice bigger (multiplied by a factor of 2).

Second, the antisymmetry factor in the case of the hypertriton equals $\sqrt{3}$. It can be calculated by the method of the second quantization given in the Appendix B of Ref. [21] that the process given by Eq. (35) has an antisymmetry factor of 2 . Therefore, the corresponding cross section is roughly estimated by scaling the cross section of the hypertriton production through

$$
\sigma\left({ }_{\Lambda}^{4} \mathrm{H}\right)=\sigma\left({ }_{\Lambda}^{3} \mathrm{H}\right) \times F=\sigma\left({ }_{\Lambda}^{3} \mathrm{H}\right) \times 4 \times \frac{4}{3} .
$$

The result is shown in Fig. 18.

\section{Conclusion}

We have investigated kaon photoproduction and electroproduction on the nucleon by means of an isobar model based on the diagrammatic technique. The transition amplitude is constructed from the background and resonant terms obtained from the appropriate Feynman diagrams. The free parameters in the model are fitted to the latest experimental data from the SAPHIR, CLAS, LEPS, and GRAAL collaborations. These data consist of differential cross sections, single and double polarization observables. The results of the present model show a substantial improvements to the predictions of Kaon-Maid.

We have also constructed a nuclear operator for photoand electroproduction of the hypertriton off the ${ }^{3} \mathrm{He}$. It is found that the effect of Fermi motion is essential in this process. The effect of the off-shell assumptions on the cross section magnitude is also significant. The assumption that the final hyperon is on-shell, while the initial nucleon is off-shell, can nicely shift the cross section upward closer to the experimental data.

We have also investigated the effects of the higher partial waves and relativistic terms on the accuracy of the calculated differential cross sections. It is shown that an accurate calculation could still be obtained if we used the three lowest partial waves with isospin zero (i.e., using $\alpha \leq 4)$ The cpu-time for calculating differential cross sections could be reduced by a factor of about 60 in this case.

Finally, we have roughly estimated the differential cross section of the ${ }_{\Lambda}^{4} \mathrm{H}$ electroproduction on the ${ }^{4} \mathrm{He}$ by use of the result on the hypertriton electroproduction. It is found that our prediction nicely fits the available experimental data.

\section{Acknowledgements}

This work has been partially supported by the University of Indonesia. 


\section{References}

1. S. Capstick and W. Roberts, Phys. Rev. D 58, 074011 (1998).

2. G. Knöchlein, D. Drechsel, and L. Tiator, Z. Phys. A 352 (1995) 327.

3. H. Haberzettl, C. Bennhold, T. Mart, and T. Feuster, Phys. Rev. C 58 (1998) R40.

4. M. Q. Tran et al., Phys. Lett. B 445 (1998) 20.

5. J.-C. David, C. Fayard, G. H. Lamot, and B. Saghai, Phys. Rev. C 53 (1996) 2613.

6. R. Bradford et al., Phys. Rev. C 73 (2006) 035202.

7. K. H. Glander et al., Eur. Phys. J. A 19 (2004) 251.

8. T. Mart and A. Sulaksono, Phys. Rev. C 74 (2006) 055203.

9. P. Bydzovsky and T. Mart, Phys. Rev. C 76 (2007) 065202.

10. T. Mart, Few Body Syst. 42, 125 (2008).

11. M. Sumihama et al., Phys. Rev. C 73 (2006) 035214.

12. R. Lawall et al., Eur. Phys. J. A 24 (2005) 275.

13. T. Mart and L. Tiator, in preparation.

14. H. Kohri et al., Phys. Rev. Lett. 97 (2006) 082003.

15. P. Ambrozewicz et al., Phys. Rev. C 75 (2007) 045203.

16. R. Nasseripour et al., Phys. Rev. C 77 (2008) 065208.

17. F. Dohrmann et al., Phys. Rev. Lett. 93 (2004) 242501.

18. L. Tiator, A. K. Rej, and D. Drechsel, Nucl. Phys. A 333 (1980) 343; L. Tiator, Nucl. Phys. A 364 (1981) 189.

19. T. Mart, Nucl. Phys. A 815 (2009) 18.

20. R. A. Adelseck, C. Bennhold and L. E. Wright, Phys. Rev. C 32 (1985) 1681.

21. T. Mart and B. I. S. van der Ventel, Phys. Rev. C 78 (2008) 014004.

22. T. Mart, L. Tiator, D. Drechsel, and C. Bennhold, Nucl. Phys. A640 (1998) 235.

23. R. A. Williams, C.-R. Ji, and S. R. Cotanch, Phys. Rev. D 41 (1990) 1449. 